\title{
Hyperglycaemic Hemichorea and Hyperglycaemic Hyperosmolar Syndrome in a Patient with HbA1c levels of $19.9 \%$ : A Rare and Potentially Reversible Case
}

\author{
Mariana Guerra, Gonçalo Silva, Daniela Marado, Jorge Fortuna, Armando Carvalho \\ Centro Hospitalar Universitário de Coimbra, Coimbra, Portugal
}

Received: 29/09/2019

Accepted: 08/10/2019

Published: 20/12/2019 How to cite this article: Guerra M, Silva G, Marado D, Fortuna J, Carvalho A. Hyperglycaemic hemichorea and hyperglycaemic hyperosmolar syndrome in a
patient with HbA1c levels of 19.9\%: a rare and potentially reversible case. EJCRIM 2019;7: doi:10.12890/2019_001305.

Conflicts of Interests: The Authors declare that there are no competing interest

This article is licensed under a Commons Attribution Non-Commercial 4.0 License

\section{ABSTRACT}

Hemichorea is a rare clinical manifestation of type 2 diabetes mellitus. The patient presents with non-ketotic hyperglycaemia, hemichorea (characterized by rapid and involuntary movements of a specific part of the body) and the CT imaging reveals the presence of alterations involving the ganglia of the base $\mathrm{e}^{[1-3]}$.

\section{LEARNING POINTS}

- Hemichorea is a rare clinical manifestation of type 2 diabetes mellitus associated with an uncontrolled disease.

- Hyperglycaemic hemichorea has a good prognosis.

- All diabetics need to maintain good metabolic control and control cardiovascular risk factors.

\section{KEYWORDS}

Diabetes mellitus, hyperglycaemic hyperosmolar syndrome, hyperglycaemic hemichorea

\section{CASE DESCRIPTION}

An 86-year-old woman, with a 20-year history of type 2 diabetes mellitus (DMT2) came to the ER due to motor agitation with involuntary movements of the left upper extremity with 3 days of evolution. She was apyretic, hypotensive and dehydrated. Involuntary, irregular and non-suppressible movements of the left superior member with involvement of the ipsilateral face were noted. The patient was found to be hyperglycaemic with a serum glucose of $1,045 \mathrm{mg} / \mathrm{dl}$ and also had hyperosmolar hyponatraemia, anaemia, leucocytosis with neutrophilia, acute renal injury and elevated troponin I, with non-supraST waves on the ECG. In the urine summary, the findings were of leucocyturia without ketonuria. Hyperglycaemic hyperosmolar syndrome and hyperglycaemic hemichorea were diagnosed. A CT scan was carried out (Fig. 1) with the identification of a hyperdensity at the level of the right striatum, which corroborated the neurological findings. Infusion with insulin therapy was started, and fluid therapy, as well as other supportive care directed towards the anaemia, acute cystitis and for the acute myocardial infarction type 2, was initiated. Antipsychotic medication was also needed. At the end of 3 days of hospitalization, the patient no longer presented involuntary movements and was discharged 6 days later. The glycated haemoglobin (HbA1c) result, however, was $19.9 \%$. 


\section{DISCUSSION}

Most patients with hyperglycaemic hemichorea have a good prognosis, provided that they maintain good metabolic control, while therapy with dopaminergic antagonists may be necessary to control chorea ${ }^{[4]}$. This is a rare and revealing manifestation of the need for a high degree of disease control, as evidenced by the HbA1c levels in our patient.

\section{REFERENCES}

1. Padmanabhan S, Zagami AS, Poynten AM. A case of hemichorea-hemiballismus due to nonketotic hyperglycemia. Diabetes Care 2013;36(4):e55-e56.

2. Cosentino C, Torres L, Nuñez Y, Suarez R, Velez M, Flores M. Hemichorea/hemiballism associated with hyperglycemia: report of 20 cases. Tremor Other Hyperkinet Mov ( $N$ Y) 2016;6:402.

3. Chang X, Hong W, Yu H, Yao Y. Chorea associated with nonketotic hyperglycemia: a case report with atypical imaging changes. Medicine 2017;96(45):e8602.

4. Ruhangisa F, Stephen H, Senkondo J, Mwasamwaja A, Kanenda S, Mbarak S, et al. Acute hemichorea in a newly diagnosed type II diabetes patient: a diagnostic challenge in resource-limited setting: a case report. BMC Res Notes 2016;9:413. 